Par 6 regul at es skel et ogenesi s and gut di fferenti at $i$ on in sea ur chi $n$ I arvae

\begin{tabular}{|l|l|}
\hline 著者 & $\begin{array}{l}\text { Shi omi Kosuke, Yamazaki At suko, Kagawa } \\
\text { M t suyoshi, K yonmt o Masat o, Yamaguchi Nasaaki }\end{array}$ \\
\hline $\begin{array}{l}\text { j our nal or } \\
\text { publ i cat i on t i t l e }\end{array}$ & Devel opment Genes and Evol ut i on \\
\hline vol une & 222 \\
\hline number & 5 \\
\hline page r ange & $269-278$ \\
\hline year & $2012-09-01$ \\
\hline URL & ht t p: //hdl . handl e. net /2297/32461 \\
\hline
\end{tabular}




\section{Par6 regulates skeletogenesis and gut differentiation in sea urchin larvae}

Kosuke Shiomi $^{1, \S}$, Atsuko Yamazaki ${ }^{1, \dagger}$, Mitsuyoshi Kagawa ${ }^{1}$, Masato Kiyomoto ${ }^{2}$, and Masaaki

$$
\text { Yamaguchi }{ }^{1, *}
$$

${ }^{1}$ Division of Life Science, Graduate School of Natural Science and Technology, Kanazawa University,

Kakuma, Kanazawa 920-1192, Japan

${ }^{2}$ Marine and Coastal Research Center, Ochanomizu University, Kouyatsu, Tateyama 294-0301, Japan

${ }^{\S}$ Present address: Department of Regenerative Medicine, National Institute for Longevity Sciences,

Gengo, Oobu 474-8522, Japan

†Present address: Research Center for Marine Biology, Tohoku University, Asamushi, Aomori 039-3501,

Japan

K. Shiomi and A. Yamazaki contributed equally to this study.

*Author for correspondence

E-mail: myama@kenroku.kanazawa-u.ac.jp

Tel: +81-76-264-6233

Fax: +81-76-264-6215 
Key words: Atypical PKC, Biomineralization, Echinoderm, PMC, Syncytial cable 


\section{Abstract}

Partitioning-defective (par) genes were originally identified as genes that are essential for the asymmetric division of the Caenorhabditis elegans zygote. Studies have since revealed that the gene products are part of an evolutionarily conserved PAR-aPKC system involved in cell polarity in various biological contexts. In this study, we analyzed the function of par6 during sea urchin morphogenesis by morpholino-mediated knockdown and by manipulation swapping of the primary mesenchyme cells (PMCs). Loss of Par6 resulted in defects in skeletogenesis and gut differentiation in larvae. Phenotypic analyses of chimeras constructed by PMC swapping showed that Par6 in non-PMCs is required for differentiation of archenteron into functional gut. In contrast, Par6 in both PMCs and ectodermal cells cooperatively regulates skeletogenesis. We suggest that Par6 in PMCs plays an immediate role in the deposition of biomineral in the syncytial cable, whereas Par6 in ectoderm may stabilize skeletal rods via an unknown signal(s). 


\section{Introduction}

Partitioning-defective (par) genes, which were originally identified in Caenorhabditis elegans, regulate asymmetric division of the zygote (Kemphues et al. 1988; Guo and Kemphues 1995; Watts et al. 1996; Tabuse et al. 1998). Subsequent studies have revealed that the gene products are part of an evolutionarily conserved PAR-aPKC system involved in cell polarity in various biological contexts. The PAR-aPKC system converts initial polarity cues in the establishment of complementary membrane domains along the polarity axis. In most cases, this is achieved by mutually antagonistic interactions between the aPKC-PAR3-PAR6 complex and PAR1 or PAR2 localized opposite (Suzuki and Ohno 2006; Goldstein and Macara 2007).

In sea urchins, the Par6-aPKC complex mediates establishment of the apicobasal polarity of eggs and early embryos via directed insertion of basolateral membrane in the cleavage furrow (Alford et al. 2009). On the other hand, Prulière et al. (2011) showed that aPKC localized at the base of cilia controls ciliogenesis in sea urchin embryos during the blastula and gastrula stages. In this study, we analyzed the function of par6 in the morphogenesis of sea urchin larvae by both morpholino-mediated knockdown and manipulation through swapping the primary mesenchyme cells (PMCs). We show that Par6 is required 
for skeletogenesis and gut differentiation in sea urchin larvae, and suggest a novel function of Par6 in the biomineralization.

\section{Materials and methods}

\section{Animals and embryos}

Adult Hemicentrotus pulcherrimus were collected near the Noto Marine Laboratory, Kanazawa

University, the Marine and Coastal Research Center, Ochanomizu University, and the Research Center for Marine Biology, Tohoku University. Gametes were collected by intracoelomic injection of $0.5 \mathrm{M} \mathrm{KCl}$.

Embryos were cultured at $15^{\circ} \mathrm{C}$ in Jamarin U artificial seawater (ASW; Jamarin Laboratory).

\section{Cloning of $\mathrm{Hp}-\mathrm{p} 16$}

Total RNA was purified using Sepasol-RNA I Super (Nacalai Tesque) and DNase I (Takara Bio Inc.). A partial fragment of Hp-p16 was isolated by PCR using GoTaq DNA Polymerase (Promega) and cDNA from embryos at the mesenchyme blastula and gastrula stages. The primers used were as follows: p16-forward, 5'-CCTTCATCGCTCTACTTGCTTTCATCGC-3' (coding for TFIALLAFIA in the signal sequence), and p16-reverse, 5'-ATACCAAACGCCATGCCGCTCTTCT-3' (coding for QKSGMAFGI in 
the transmembrane domain and the N-terminal flanking region). The 3'-fragment of Hp-p16 cDNA was

obtained by RACE using the GeneRacer Kit (Invitrogen), p16-forward primer (described above), and

KOD FX DNA Polymerase (TOYOBO). The PCR products were cloned into the pTA2 vector

(TOYOBO) and sequenced using the Genetic Analyzer 3100 (Applied Biosystems) with the Big Dye

Terminator v3.1 Cycle Sequencing Kit (Applied Biosystems). Predicted Hp-P16 sequence was 96\%

identical to residues 13-173 of Sp-P16 (Accession No. NM_214646). The sequence has been deposited in the DNA Data Bank of Japan as Hp-p16 (Accession No. BAK74802).

\section{Preparation of constructs used for in vitro transcription}

Modified pBluescript RN3 (pBluescript RN3'; Nishimura et al. 2004) was used to make expression

constructs. For rescue experiments, a truncated construct lacking the $21 \mathrm{bp}$ following the initiation

methionine codon was generated by inverse-PCR, using Hp-par6 in pBluescript RN3' (Shiomi and

Yamaguchi 2008) and KOD FX DNA polymerase (TOYOBO). The primers used were as follows:

Truncate-par6-forward, 5'-GCGAAAAATGGATCCACGACAAT-3'; and Truncate-par6-reverse,

5'-CATTTGAAGCGGGAAATCATCAAT-3'. We confirmed the sequence of the construct before

transcription. 


\section{Microinjection}

The morpholino oligonucleotide (Mpar6) and a standard control oligo were obtained from Gene Tools.

The sequence and positions of Mpar6 with respect to the translation initiation site were as follows:

5’-CCATGATGCCCACCGAGTCCCGCAT-3’ (+1 to +25). Capped RNA was transcribed from a linearized template plasmid using mMessage mMachine T3 Kit (Ambion) according to the manufacturer's protocol. RNA lacking an Mpar6-target site was diluted to $3.3 \mathrm{pg} / \mathrm{pl}$ in 40\% glycerol; 5 pl of each solution was injected into fertilized eggs as described by Gan et al. (1990).

\section{Microsurgery}

Before microsurgery, the embryos were treated with double-strength Jamarin U for 1-2 min to stop their swimming and placed in micromanipulation chambers filled with ASW. Micromanipulation to remove PMCs was carried out according to the method of Ettensohn and McClay (1988). A microneedle loaded with ASW was inserted into the blastocoel of a mesenchyme blastula at $24 \mathrm{~h}$, and PMCs were washed away by a stream of ASW. In PMC-swapping experiments, donor embryos were stained with $5 \mu \mathrm{g} / \mathrm{ml}$ rhodamine B isothiocyanate (RITC; Research Organic Inc.) for 1.5 h, after which a microneedle was 
inserted into the blastocoel and PMCs were sucked out. After PMCs were collected from two or three embryos, approximately 60 PMCs were injected into the blastocoel of one host embryo.

\section{RT-PCR}

To estimate the expression levels of marker genes in embryos at the mesenchyme blastula stage, cDNA was synthesized from total RNA using ReverTra ACE (Toyobo) and random 9mer oligonucleotides. PCR was carried out using ThermoStart Taq DNA polymerase (ABgene). For each PCR reaction, cDNAs derived from 0.2 and 0.004 embryos were used to amplify markers and ubiquitin, respectively. Primer sequences were as follows: delta-F, 5'-GTACGTGTCGCAATGAAG-3'; delta-R, 5'-AACAGTGGTCACGGATCT-3'; sm50-F, 5'-GGCTAGTATTGTAGCCTT-3'; sm50-R, 5'-GGCGAATCCGTTAGGATA-3'; endo16-F, 5'-GCAACTTCCGATCATGTTGT-3'; endo16-R, 5'-GCGATTCTCCTTGTACTC-3'; ubiquitin-F, 5'-CTCACAGGCAAGACCAT-3'; and ubiquitin-R, 5'-GTCTCTCAATTTGTCTATAGCT-3'. 
WMISH was performed according to the method of Minokawa et al. (2004) for par6 or to that of Hibino

et al. (2004) for sm30, p16, and apkc. Digoxygenin (DIG)-labeled probes were transcribed using

DIG-labeling mix (Roche) from Hp-apkc, par6 (Shiomi and Yamaguchi 2008), sm30 (Kitajima et al.

1996), and Hp-p16 cDNAs (this study).

\section{Antibodies and whole-mount immunostaining}

For immunostaining, we fixed embryos and larvae according to the method of Hibino et al. (2004). Fixed

embryos were washed with PBS containing 0.1\% Triton X-100 (PBT), blocked with 2\% bovine serum

albumin (BSA), and then incubated for $2 \mathrm{~h}$ with goat polyclonal anti-PAR6 antibody (diluted 1:100 with

PBT; Santa Cruz, sc-14405; Alford et al. 2009), the B2C2 monoclonal antibody (diluted 1:500 with PBT;

Leaf et al. 1987), or the P4 monoclonal antibody (diluted 1:200 with PBT; Shimizu et al. 1988). Although

the reactivity of the P4 antibody with PMCs is quite similar to that of the B2C2 antibody,

Shimizu-Nishikawa et al. (1990) showed that these antibodies react with the same group of glycoproteins,

including MSP130, but their epitopes are different. Alexa Fluor 488-conjugated anti-goat IgG or

anti-mouse IgG (Molecular Probes) was used as the secondary antibody. 


\section{Results}

Par6 is accumulated along skeletal rods and in digestive tract cells in larvae

The distribution of Par6 protein in H. pulcherrimus larvae was determined using an antibody, which specifically recognizes Par6 in a variety of vertebrate and sea urchin species (Alford et al. 2009).

Although par6 is ubiquitously expressed during the development (Shiomi and Yamaguchi 2008; Fig. 1a-e), in pluteus larvae, Par6 signals were detected along the skeletal rods (Fig. 1i-k); Par6 appeared to be localized in the syncytial cable of PMCs, since few signals were observed in the PMC cell body or spicule space, where calcite had been dissolved during fixation (asterisk and inset in Fig. 1i; also see Discussion). In addition, Par6 was distributed in endodermal cells forming the boundary between the midgut and hindgut (arrows in Fig. 1f,g,i,j), and cells encircling the anus (arrowheads in Fig. 1h,k).

We injected a morpholino oligo against par6 mRNA (Mpar6) into fertilized eggs. Larvae including Mpar6 did not exhibit the Par6 accumulations observed in controls (Fig. 2o,p), indicative of the efficacy of Mpar6 on translational inhibition.

Par6 is required for skeletogenesis and gut differentiation 
Mpar6-injected zygotes cleaved normally, including unequal fourth cleavages that formed micromeres,

and developed into mesenchyme blastulae via swimming blastulae (Fig. 2a,h). Similar to controls,

embryos including Mpar6 (Mpar6 embryos) expressed early marker genes, including delta (presumptive

secondary mesenchyme cell), sm50 (PMC), and endo16 (endomesoderm), indicating that early

endomesoderm specification occurred in Mpar6 embryos (Fig. 3a).

Morphological defects in Mpar6 embryos emerged after gastrulation during two processes:

skeletogenesis and gut differentiation. When controls developed into prism larvae at 48 h (Fig. 2c,d),

Mpar6 embryos formed spot-like or triradial spicule rudiments within the ventrolateral PMC clusters (Fig.

2j,k). However, subsequent growth of the skeleton was severely impaired (Fig. 2m); the spicule largely

remained as it was in Mpar6 embryos at $48 \mathrm{~h}$. We note that Mpar6 embryos, especially after gastrulation,

had a thicker ectoderm wall than uninjected controls, which reduced the size of the experimental

embryo/larva (Fig. 2i,j,l).

The larval skeleton is elaborated through interactions between PMCs and ectodermal cells

(Armstrong et al. 1993; Ettensohn and Marinda 1993; Guss and Ettensohn 1997; Hardin and Armstrong

1997). Duloquin et al. (2007) demonstrated that localized VEGF acts as both a guidance cue for the

formation of ventrolateral PMC clusters and a signal for the differentiation of skeletogenic cells. On the 
other hand, Röttinger et al. (2008) showed that FGF signaling plays an essential role in the guidance of

PMC migration and the direction of skeletal morphogenesis. However, defects in skeletogenesis induced by Mpar6 injection were unlikely due to either the loss of skeletogenic cell differentiation or a lack of ectodermal guidance/direction cues required for skeletogenesis, since in Mpar6 embryos, mesenchyme cells expressed the PMC-specific P4 antigen (Fig. 3j; Shimizu et al. 1988) and VEGF/FGF-dependent skeletogenic genes, such as sm50 and sm30 (Fig. 3a,m; Duloquin et al. 2007; Röttinger et al. 2008), and because their descendants expressing the B2C2 antigen (Fig. 31; Leaf et al. 1987) were allocated on the inner ectoderm wall in a pattern that prefigures the rod branching patterns, i.e., the anterolateral, transverse, postoral, and body rods (Fig. 3l). Furthermore, skeletogenic cells expressed $p 16$ that is essential for skeletal rod elongation (Fig. 3n,o; Cheers and Ettensohn 2005). However, the size of the PMC ring in Mpar6 larvae was smaller than in controls, probably due to dwarfish ectoderm patterning and/or a lack of accompanying growth of skeletal rods in the experimental larvae.

Another defect in Mpar6 larvae was lack of gut differentiation. Although gastrulation was delayed for several hours, Mpar6 embryos developed archenteron, from the tip of which the secondary mesenchyme cells (SMCs) had been delaminated (Fig. 2i,j). Subsequently, Mpar6 larvae developed SMC-derived tissues, such as pigment cells (Fig. S1a), circumesophageal muscle cells, and coelomic 
pouches, although the number of pigment cells was lower than in controls in some batches. In contrast,

the larva did not develop a fully differentiated gut in either morphology or function; the segmentation of

archenteron was repressed, and the mid/hindgut region lacked alkaline phosphatase activity (Fig. 2n).

Co-injection of par6 mRNA without the Mpar6-target sequence ( 15 pg/egg) rescued Mpar6

larvae from defects in skeletogenesis and gut differentiation, but partially in ectoderm patterning.

Co-injected zygotes developed into pluteus larvae with a slightly small skeleton, but normal in pattern

(Fig. 2r,u) as well as tripartite gut with alkaline phosphatase activity (Fig. 2s,v). However, the larva was

smaller and distinct from control larvae in shape (arrowhead in Fig. 2q). In contrast, zygotes injected with

mRNA alone developed normally (Fig. S1c,d). These observations indicate a requirement for Par6 for

skeletogenesis and gut differentiation in sea urchin larvae; they also suggest that higher and/or longer

doses of Par6 may be required for correct ectoderm patterning, since co-injection of a lower dose of

mRNA ( 10 pg/egg) rescued morphants from defects in skeletogenesis and gut differentiation, but not

from dwarfish ectoderm patterning (Fig. S1b,b’).

\section{Par6 controls skeletogenesis in PMCs and ectoderm cooperatively}

Skeletogenesis in sea urchin larvae is controlled by both PMCs and ectodermal cells (Armstrong et al. 
1993; Ettensohn and Marinda 1993; Guss and Ettensohn 1997; Hardin and Armstrong 1997; Duloquin et al. 2007; Röttinger et al. 2008). On the other hand, Hamada and Kiyomoto (2003) demonstrated that unidentified signals from PMCs regulate gut differentiation. When PMCs are deleted from the mesenchyme blastula, both the constrictions in archenteron and the expression of alkaline phosphatase are delayed. To clarify whether defects in skeletogenesis and gut differentiation in par6 morphants are due to loss of Par6 in PMCs or the rest of embryos, or both, we carried out PMC-swapping experiments using mesenchyme blastulae at $24 \mathrm{~h}$ after fertilization.

Sham controls, which consisted of a PMC-deleted host embryo and PMCs transplanted from sibling rhodamine-labeled embryos, developed into pluteus larvae indistinguishable from the unmanipulated controls (Fig. 4a-f), although one and two of the 13 chimeras had an abnormal skeletal pattern and extra rods, respectively (Table 1). All transplanted PMCs examined were arranged along the skeletal rods in the chimeras. To examine the effects of morpholino injection on eggs we constructed chimeric embryos composed of a PMC-deprived host and PMCs including a control morpholino, and confirmed that their phenotypes were practically identical to those of the sham control (3/3 cases, data not shown). Thus, we analyzed the phenotypes of embryos with Mpar6 present only in PMCs (Fig. 4g-l). The chimera developed into gastrula-like larvae with spicule rudiments on two ventrolateral positions at $48 \mathrm{~h}$ 
(24 h after manipulation; Fig. 4h,h'; 9/11 cases). However, skeletal rod elongation from the rudiments

was delayed in the chimera, although PMC descendants were arranged in a ring pattern with two clusters plus two cables that will form the anterolateral rods, like in control chimeras (Fig. 4h,i). This defect in skeletogenesis continued during the next $24 \mathrm{~h}$; the skeleton remained stunted, and the branching pattern was irregular (Fig. 4k; Fig. S2; Table 1). When compared to spicules in par6 whole morphants (Fig. 2m), however, restoration of skeletal rod elongation was evident in the chimera (Fig. 4k; Fig. S2). This observation suggests that Par6 cooperatively regulates skeletogenesis in both PMCs and the rest of the embryo (probably in the ectoderm). Noticeably, the chimera lacked a connection of bilateral body rods at the vertex (Fig. 4k'; Fig. S2; Table 1), although PMC descendants were correctly allocated in the region (arrowhead in Fig. 4l'). In contrast, the chimera developed a tripartite gut with alkaline phosphatase activity (Fig. 4t; Table 1), indicating that Par6 is not involved in gut differential signals from PMCs.

\section{Par6 in non-PMCs regulates gut differentiation}

Next, we analyzed the phenotypes of complementary chimeras composed of a PMC-less host including Mpar6 and normal PMCs. Like par6 whole morphants (Fig. 2i, j, l), the chimera had a thicker ectoderm wall and was therefore smaller than control or complementary chimeras (Fig. 4m,p), supporting the 
notion that Par6 is required for correct ectoderm patterning. At $48 \mathrm{~h}$, some chimeras generated extra small spicules in addition to two spicules in the ventrolateral PMC clusters (Fig. 4n'; 4/6 cases); the latter two constituted most of the larval skeletal rods, while the former gave rise to small spines on the body rods (see A1 and B3 in Fig. S3). Unlike complementary chimeras, the chimera formed a connection of bilateral body rods at the vertex (Fig. 4q,q'; Fig. S3; Table 1). In contrast, skeletal rod elongation was retarded in the oral side of chimeras (Fig. 4q,q'; Fig. S3; Table 1); the defect was evident in both the postoral rods and anterolateral rods toward the oral hood, when compared to those of complementary chimeras (Fig. 4k,k’; Fig. S3). These observations not only support the notion that Par6 cooperatively regulates skeletogenesis in PMCs and ectoderm, but also indicate that ectoderm produces an unidentified Par6-dependent signal(s) that is necessary for skeletal rod elongation particularly in the oral field. On the other hand, the chimera did not develop tripartite gut with alkaline phosphatase activity (Fig. 4u; Table 1), indicating that Par6 in non-PMCs is required for gut differentiation. Distribution of Par6-accumulating cells in the digestive tract (Fig. 1f-k) suggests that Par6 may control gut differentiation via the apical constriction. 
To examine whether loss of Par6 affects the apkc expression, we analyzed the apkc expression in par6

morphants. In controls, zygotic apkc expression starts in the vegetal plate, followed by expression

restricted to PMCs and the archenteron tip during gastrulation (Fig. 5a,b), and finally, the expression

fades by the late gastrula stage. From the prism stage, the second phase of apkc expression starts in three

cell types: ectodermal cells interspersed in the ciliary band, skeletogenic cells at the vertex, and stomach

cells (Fig. 5c). The apkc-expressing cells in the ciliary band do not appear to match the serotonergic cells

(four to six cells in the apical ganglion; Bisgrove and Burke 1986) or synaptotagmin-expressing cells

(flask-shaped cell bodies on the basal side of ectoderm; Nakajima et al. 2004), although apkc-expressing

cells may partially overlap the neural cells. In par6 morphants, the first and second phases of apkc

expression occurred as they do in controls (Fig. $5 d-f$ ), except for the expression in ectodermal cells in the

oral hood (arrowhead in Fig. 5f). This apkc downregulation induced by loss of Par6 is probably indirect,

since Par6 is not expressed in the ciliary band of larvae (Fig. 20), and because dwarfish ectoderm

patterning at earlier stages is a constant phenotype observed in par6 morphants (Fig. 2i; Fig. 3l) and

chimeras with loss of Par6 in non-PMC tissues (Fig. 4m). Defects of the chimera in skeletal rod

elongation in the oral field may be due to the apkc downregulation, since the defect is a unique phenotype

of chimeras with loss of Par6 in non-PMC tissues, including ectoderm. 


\section{Discussion}

\section{Par6 may function outside the polarity complex}

Par6 is a component of the apical polarity complex that contains Par3 and aPKC (Suzuki and Ohno 2006;

Goldstein and Macara 2007). Defects in par6 morphants or chimeric larvae, i.e., retardation of skeletal

rod elongation and gut differentiation, largely coincide with zygotic apkc domains (Shiomi and

Yamaguchi 2008; Fig. 5a-c). Prulière et al. (2011) have shown the aPKC expression in Paracentrotus

larvae by immunostaining; aPKC is distributed in the ciliary band ectoderm and stomach endoderm, but

not in PMCs or syncytial cables. We confirmed that the expression pattern is essentially conserved in

Hemicentrotus larvae (Fig. S4). The lack of co-expression of Par6 and aPKC in the syncytial cable or

ciliary band suggests that Par6 or aPKC may control skeletogenesis outside the polarity complex.

In Hemicentrotus larvae, however, skeletogenic cells at the vertex exhibit relatively stronger

aPKC signals than other skeletogenic cells (Fig. S4b’,e). This expression may support Par6 function as

the polarity complex in skeletogenesis, since one of the definite roles of Par6 in PMCs appears to

generate a connection of bilateral body rods at the vertex (Fig. 4k’; Fig. S2; lack of the skeletal element is

a unique phenotype of chimeras with loss of Par6 only in PMCs), and because skeletogenic cells at the 
vertex exclusively express apkc (arrow in Fig. 5c). This in turn implies that distinct mechanisms may

work in skeletogenesis between the oral and aboral sides of larvae. Alternatively, aPKC activity is

required only for the initial step of subcellular localization processes of Par6 into the syncytial cable,

since $a p k c$ is transiently expressed in PMCs (Fig. 5). Furthermore, the aPKC expression pattern is

puzzling; the protein is distributed throughout the ciliary band (Fig. S4), but the mRNA is restricted to

isolated cells in this band (Fig. 5c). The pattern suggests that apkc expression may oscillate in individual

cells in the band. To examine the aPKC function in skeletogenesis, we carried out morpholino-mediated

apkc knockdown; however, it resulted in embryonic lethality at the blastula stage (Shiomi and Yamaguchi, unpublished).

\section{Par6 function in PMCs}

Spicule formation takes place in a membrane-delineated compartment (vacuole) enclosed in the syncytial

cable formed by fusion of filopodia of PMCs (Okazaki 1960). Spicule matrix proteins, such as SM30 and

SM50, are tethered to calcite deposited in the vacuole (Seto et al., 2004), whereas MSP130 and P16 are

localized on the surface of PMCs and their filopodia (Anstrom et al. 1987; Cheers and Ettensohn 2005).

In contrast, Par6 seems to be accumulated in the cortex and/or cytoplasm of the syncytial cable (Fig. 
1f-k), although further examination is needed to define its precise localization. Loss of Par6 causes

defects in the skeletal rod elongation, but practically no effect on expressions of spicule matrix proteins or related genes (Fig. 3), suggesting that Par6 may play a more immediate role in the deposition of biomineral. Like Par6, P16 acts at relatively late step in skeletogenesis; P16 is not required for PMC specification, ingression, migration, or fusion, but essential for skeletal rod elongation (Cheers and Ettensohn 2005). Cheers and Ettensohn (2005) suggest that P16 may be a receptor for ectoderm-derived signals required for skeletal rod elongation; alternatively, P16 may function in the plasma membrane or an intracellular membrane compartment to regulate calcium transport. Since phenotypes of $p 16$ knockdown resemble those of chimeric embryos with Mpar6 present only in PMCs, it seems possible that Par6 relays signals transduced by P16 or cooperates with P16 to deposit biomineral.

\section{Par6 function in ectoderm}

From phenotype comparisons between whole par6 morphants and chimeric larvae (Fig. 2m; Fig. 3e,k,q),

it is evident that ectoderm emanates a Par6-dependent signal(s) that promotes skeletogenesis. In chimeras with loss of Par6 in PMCs, skeletal rods are often irregular in branching pattern or in shape (Fig. 4k; Fig. S2; Table 1), whereas no abnormal branching pattern is observed in chimeras with loss of Par6 in 
non-PMCs (Fig. 4q; Fig. S3; Table 1). Furthermore, zygotes injected with par6 mRNA develop into larvae with normal skeleton (Fig. S1c,d). These observations suggest that, unlike VEGF or FGF, the ectoderm-derived signal does not act as positional or directional guidance cues in skeletogenesis, but appears to maintain or stabilize spicule materials that have been deposited in the vacuole. This may explain observations that loss of the signal decreases skeletal rods in size in whole morphants compared to chimeras with loss of Par6 in PMCs (Fig. 2m; Fig. 4k) and that Par6 does not appear to be localized in ectoderm overlying growing skeletal rods (Fig. 20).

\section{Biomineralization in echinoderms}

Biomineralized tissues are considered to have arisen independently in vertebrates and echinoderms

(Bottjer et al. 2006; Livingston et al. 2006). Their matrix proteins may be an example of evolutionary

convergence. Secreted proteins associated with tooth and bone in vertebrates are believed to have arisen from an ancestral gene encoding secreted protein that is acidic and cysteine-rich (Kawasaki and Weiss 2006). In contrast, many of the secreted biomineralization proteins in echinoderms probably arose from an ancestral C-lectin domain-containing protein (Livingston et al. 2006). On the other hand, aPKC is involved in at least two independent signaling cascades that control cell polarity and cell survival; in the 
cascades, aPKC function is modulated by different partner proteins, Par6 and p62, respectively (Moscat and Diaz-Meco 2000). In biomineralization of mice, p62 is an essential mediator of aPKC function during osteoclastogenesis (Durán et al. 2004), while Par6 regulates skeletogenesis in sea urchin larvae; however, the requirement of aPKC function for skeletogenesis remains to be clarified. Further studies will reveal unique molecular mechanisms of biomineralization in sea urchins, which may reveal another role of Par6 and/or aPKC.

\section{Acknowledgments}

We thank Hideki Katow, Rudolf A. Raff, and Takashi Yamamoto for providing the P4, B2C2 monoclonal antibodies, and sm30 cDNA, respectively. We also thank staff of the Research Center for Marine Biology,

Tohoku University, for supplying adult sea urchins. We thank the reviewers whose thoughtful suggestions helped us to improve the manuscript. This study was supported by JSPS KAKENHI Nos.

06J00270 and 21570222 to AY and MY, respectively. 


\section{Figure legends}

Fig. 1 Distributions of par6 mRNA and Par6 protein. a-e Expressions of par6 analyzed by WMISH. This gene is ubiquitously expressed in the egg (a), 32-cell stage embryo (b), mesenchyme blastula (c), gastrula (d), and pluteus larva (e). f-k Distributions of Par6 in larvae (72 h) analyzed by immunostaining. f, i Ventral views of aboral half of a larva. $\mathbf{g}, \mathbf{h}, \mathbf{j}, \mathbf{k}$ Ventrolateral views. Signals are distributed along skeletal rods (i-k); Par6 seems to be localized in the syncytial cable of PMCs, since few signals are observed in the PMC cell body or spicule space, where calcite has been dissolved during fixation (asterisk and inset in i). In addition, signals are detected in endodermal cells forming the mid-hindgut boundary (arrows in $\mathbf{f}, \mathbf{g}, \mathbf{i}, \mathbf{j}$ ) and cells encircling the anus/blastopore (arrowheads in $\mathbf{h}, \mathbf{k}$ ). Insets in $\mathbf{i}, \mathbf{j}$, and $\mathbf{k}$ show high-magnification images around the asterisk, arrow, and arrowhead regions, respectively.

Fig. 2 Par6 is required for skeletogenesis and gut differentiation in sea urchin larvae. a-g Control embryos and larvae. h-n Embryos and larvae derived from zygotes injected with Mpar6. Embryos including Mpar6 developed almost normally up to the mesenchyme blastula stage (a, h). Although there was a delay in gastrulation for several hours, the embryo developed into gastrulae, which had a thicker ectoderm wall, and were thus smaller than controls (b, i). In larvae including Mpar6, growth of the 
skeletal rods was severely impaired, although spicule rudiments formed within the ventrolateral PMC

clusters ( $\mathbf{k}, \mathbf{m})$. In addition, the larva did not develop a fully differentiated gut in either morphology or

function (l, n); growth of the midgut and segmentation of archenteron were repressed $(\mathbf{l}, \mathbf{n})$, and the

mid/hindgut region lacked alkaline phosphatase activity (n). o, p Par6 distributions of control and Mpar6

larvae. In a control larva (o), Par6 is accumulated in syncytial cables (asterisk), endodermal cells (arrow),

and cells around blastopore (arrowhead), whereas a larva including Mpar6 does not exhibit either of the

accumulations (p). $\quad$ q-v Larvae including Mpar6 and par6 mRNA without Mpar6 target sequence.

Co-injected zygotes developed into pluteus larvae with a slightly smaller skeleton, but normal in pattern

$(\mathbf{q}, \mathbf{r}, \mathbf{t}, \mathbf{u})$, as well as tripartite gut with alkaline phosphatase activity (s, v). Inset in $\mathbf{s}$ shows a

high-magnification image around the mid/hindgut region, where arrowhead indicates the hindgut with

alkaline phosphatase activity. However, the larva was distinct from control larvae in shape; the

posterodorsal side of the aboral ectoderm wall was fallen (arrowhead in $\mathbf{q}$ ), when compared to controls

(e). j, k, n, v Dorsal views. o, p, t, u Ventral views

Fig. 3 Morpholino-mediated par6 knockdown did not affect the expression of early endomesoderm

specification genes or the differentiation of the skeletogenic cell. a The expressions of marker genes in 
embryos including Mpar6. Total RNA was extracted from control embryos and embryos including Mpar6

at the mesenchyme blastula stage ( $24 \mathrm{~h}$ ). The expression of marker genes delta (presumptive SMC), sm50

(PMC), endo16 (endomesoderm), and ubiquitin (ubiquitous) was analyzed by RT-PCR. Numerals below indicate the number of PCR cycles. Expression levels of these genes in embryos including Mpar6 were comparable to those in control embryos. b-e, $\mathbf{i}-\mathbf{l}$ Expression of the P4 (b-e) and B2C2 antigens (i-l) analyzed by immunostaining. In either controls (b-e) or par6 morphants (i-l), PMCs expressing the P4 antigen (b, c, i, j) ingressed at $24 \mathrm{~h}$, and skeletogenic cells expressing B2C2 antigen (d, e, k, l) were allocated on the inner ectoderm wall in a pattern that prefigures the rod-branching patterns in larvae at 48 h. However, the PMC-ring in par6 morphants was smaller than that in controls $(\mathbf{e}, \mathbf{l}) . \mathbf{f}-\mathbf{h}, \mathbf{m}-\mathbf{o}$ Expressions of sm30 (f, m) and $p 16$ (g, h, n, o) analyzed by whole-mount in situ hybridization (WMISH). Like in controls (f), sm30 was expressed in skeletogenic cells in the par6 morphant (m). In either controls or par6 morphants, p16 was expressed in PMCs at the mesenchyme blastula stage (26 h; $\mathbf{g}, \mathbf{n})$. At the pluteus stage (72 h), the expression was restricted to PMCs at sites of skeletal rod elongation, such as the tips of the postoral, anterolateral, and body rods (h, o), although skeletal rod elongation was severely impaired in par6 morphants (o). d-f, $\mathbf{k}-\mathbf{m}$ Dorsal views. g, $\mathbf{n}$ Vegetal views. h, $\mathbf{o}$ Ventral views 
Fig. 4 Development of chimeric embryos constructed by PMC swapping. Illustrations on the left indicate combinations of a PMC-less host (upper, from normal or Mpar6-injected embryos) and transplanted PMCs (lower in parentheses, from normal or Mpar6-injected embryos), in which cells including Mpar6 are blue. a-u Lateral views. a'-r' Ventral views. a-f, s Control chimeras composed of a PMC-less host and transplanted PMCs labeled with rhodamine. The chimera developed normally like unmanipulated controls. g-l, t Chimeras composed of a PMC-less host and PMCs including Mpar6. Although PMCs were arranged in a ring pattern with two ventrolateral clusters in the chimera, rod elongation from spicule rudiments was delayed (48 h; h, h'), and the branching pattern was irregular in most larvae (72 h; k, k'). Note that the chimera lacked a connection of bilateral body rods at the vertex ( $\left.\mathbf{k}^{\prime}\right)$, in spite of correct allocation of skeletogenic cells in the region (arrowhead in l'). Arrowhead in $\mathbf{l}$ indicates rhodamine-positive cells in the gut region. The chimera developed a tripartite gut with alkaline phosphatase (AP) activity (t). m-r, u Chimeras composed of a PMC-less host including Mpar6 and normal PMCs. The chimera had a thicker ectoderm wall $(\mathbf{m}, \mathbf{p})$ and thus was smaller than control chimeras (a, d). At $48 \mathrm{~h}$ (24 h after manipulation), some chimeras generated extra small spicules (small arrowheads in n') in addition to two spicules in ventrolateral PMC clusters (large arrowheads in n'). The latter two constituted most of the larval skeletal rods at $72 \mathrm{~h}(\mathbf{q})$. Note that rod elongation was retarded in 
the oral field, whereas the connection of bilateral body rods was formed at the vertex (q'). Arrowhead in $\mathbf{r}$ indicates rhodamine-positive cells in the gut region. The chimera did not develop a functional gut (u), unlike complementary chimeras (t)

Fig. 5 Expression of apkc in par6 morphants analyzed by WMISH. a, b, d, e Lateral views. c, f Dorsal views. a-c Control embryos and larvae. The first phase of zygotic apkc expression starts in the vegetal plate at the blastula stage (a). Expression is maintained in PMCs and the archenteron tip during gastrulation (b), but fades at the late gastrula stage. During the prism to pluteus stages, the second phase of expression starts in three cell types (c): ectodermal cells interspersed in the ciliary band, skeletogenic cells at the vertex (arrow in c), and stomach cells. d-f Embryos and larvae including Mpar6. The first and second phases of apkc expression occurred in par6 morphants as they did in controls (d-f), except for expression in ectodermal cells in the oral hood (arrowhead in $\mathbf{f}$ )

\section{References}

Alford LM, Ng MM, Burgess DR (2009) Cell polarity emerges at first cleavage in sea urchin embryos.

Dev Biol 330:12-30. doi:10.1016/j.ydbio.2009.02.039 
Anstrom JA, Chin JE, Leaf DS, Parks AL, Raff RA (1987) Localization and expression of msp130, a primary mesenchyme lineage-specific cell surface protein in the sea urchin embryo. Development 101:255-265

Armstrong N, Hardin J, McClay DR (1993) Cell-cell interactions regulate skeleton formation in the sea urchin embryo. Development 119:833-40

Bidgrove BW, Burke RD (1986) Development of serotonergic neurons in embryos of the sea urchin, Strongylocentrotus purpuratus. Dev Growth Differ 28:569-74.

doi:10.1111/j.1440-169X.1986.00569.x

Bottjer DJ, Davidson EH, Peterson KJ, Cameron RA (2006) Paleogenomics of echinoderms. Science 314:956-960. doi:10.1126/science.1132310

Cheers MS, Ettensohn CA (2005) P16 is an essential regulator of skeletogenesis in the sea urchin embryo.

Dev Biol 283:384-396. doi:10.1016/j.ydbio.2005.02.037

Duloquin L, Lhomond G, Gache C (2007) Localized VEGF signaling from ectoderm to mesenchyme cells controls morphogenesis of the sea urchin embryo skeleton. Development:2293-2302. doi:10.1242/dev.005108

Durán A, Serrano M, Leitges M, Flores JM, Picard S, Brown JP, Moscat J, Diaz-Meco MT (2004) The 
atypical PKC-interacting protein p62 is an important mediator of RANK-activated

osteoclastogenesis. Dev Cell 6:30-309. doi:10.1016/S1534-5807(03)00403-9

Ettensohn CA, McClay DR (1988) Cell lineage conversion in the sea urchin embryo. Dev Biol

125:396-409. doi:10.1016/0012-1606(88)90220-5

Ettensohn CA, Malinda KM (1993) Size regulation and morphogenesis: a cellular analysis of

skeletogenesis in the sea urchin embryo. Development 119:155-167

Gan L, Zhang W, Klein WH (1990) Repetitive DNA sequences linked to the sea urchin spec genes

contain transcriptional enhancer-like elements. Dev Biol 139:186-196.

doi:10.1016/0012-1606(90)90287-S

Goldstein B, Macara IG (2007) The PAR proteins: fundamental players in animal cell polarization. Dev

Cell 13:609-622. doi:10.1016/j.devcel.2007.10.007

Guo S, Kemphues KJ (1995) par-1, a gene required for establishing polarity in C. elegans embryos,

encodes a putative Ser/ Thr kinase that is asymmetrically distribution. Cell 81:611-620.

doi:10.1016/0092-8674(95)90082-9

Guss KA, Ettensohn CA (1997) Skeletal morphogenesis in the sea urchin embryo: regulation of primary

mesenchyme gene expression and skeletal rod growth by ectoderm-derived cues. Development 
124:1899-1908

Hamada M, Kiyomoto M (2003) Signals from primary mesenchyme cells regulate endoderm

differentiation in the sea urchin embryo. Dev Growth Differ 45:339-350.

doi:10.1046/j.1440-169X.2003.00702.x

Hardin J, Armstrong N (1997) Short-range cell-cell signals control ectodermal patterning in the oral region of the sea urchin embryo. Dev Biol 182(1):134-49. doi:10.1006/dbio.1996.8436

Hibino T, Harada Y, Minokawa T, Nonaka M, Amemiya S (2004) Molecular heterotopy in the expression of Brachyury orthologs in order Clypeasteroida (irregular sea urchins) and order Echinoida (regular sea urchins). Dev Genes Evol 214:546-558. doi:10.1007/s00427-004-0437-x

Kawasaki K, Weiss KM (2006) Evolutionary genetics of vertebrate tissue mineralization: the origin and evolution of the secretory calcium-binding phosphoprotein family. J Exp Zool B Mol Dev Evol 15:295-316. doi:10.1002/jez.b.21088

Kemphues KJ, Priess JR, Morton DG, Cheng NS (1988) Identification of genes required for cytoplasmic localization in early C. elegans embryos. Cell 52:311-320. doi:10.1016/S0092-8674(88)80024-2

Kitajima T, Tomita M, Killian CE, Akasaka K, Wilt FH (1996) Expression of spicule matrix protein gene SM30 in embryonic and adult mineralized tissues of sea urchin Hemicentrotus pulcherrimus. Dev 
Growth Differ 38:687-695. doi:10.1046/j.1440-169X.1996.t01-5-00012.x

Leaf DS, Anstrom, JA, Chin JE, Harkey MA, Showman RM, Raff RA (1987) Antibodies to a fusion protein identify a cDNA clone encoding msp130, a primary mesenchyme-specific cell surface protein of the sea urchin embryo. Dev Biol 121:29-40. doi:10.1016/0012-1606(87)90135-7

Livingston BT, Killian CE, Wilt F, Cameron A, Landrum MJ, Ermolaeva O, Sapojnikov V, Maglott DR, Buchanan AM, Ettensohn CA (2006) A genome-wide analysis of biomineralization-related proteins in the sea urchin Strongylocentrotus purpuratus. Dev Biol 300:335-348.

doi:10.1016/j.ydbio.2006.07.047

Minokawa T, Rast JP, Arenas-Mena C, Franco CB, Davidson EH (2004) Expression patterns of four different regulatory genes that function during sea urchin development. Gene Expr Patterns 4:449-456. doi:10.1016/j.modgep.2004.01.009

Moscat J, Diaz-Meco MT (2000) The atypical protein kinase Cs. Functional specificity mediated by specific protein adapters. EMBO Rep 1:399-403. doi:10.1093/embo-reports/kvd098 Nakajima Y, Kaneko H, Murray G, Burke RD (2004) Divergent patterns of neural development in larval echinoids and asteroids. Evol Dev 6:95-104. doi:10.1111/j.1525-142X.2004.04011.x 
Nishimura Y, Sato T, Morita Y, Yamazaki A, Akasaka K, Yamaguchi M (2004) Structure, regulation, and function of micro1 in the sea urchin Hemicentrotus pulcherrimus. Dev Genes Evol 214:525-536. doi:10.1007/s00427-004-0442-0

Okazaki K (1960) Skeleton formation of sea urchin larvae II. Organic matrix of the spicule. Embryologia 5:283-320. doi:10.1111/j.1440-169X.1960.tb00096.x

Prulière G, Cosson J, Chevalier S, Sardet C, Chenevert J (2011) Atypical protein kinase C controls sea urchin ciliogenesis. Mol Biol Cell 22:2042-53. doi:10.1091/mbc.E10-10-0844

Röttinger E, Saudemont A, Duboc V, Besnardeau L, McClay D, Lepage T (2008) FGF signals guide migration of mesenchymal cells, control skeletal morphogenesis and regulate gastrulation during sea urchin development. Development 135:353-65. doi:10.1242/dev.014282

Seto J, Zhang Y, Hamilton P, Wilt F (2004) The localization of occluded matrix proteins in calcareous spicules of sea urchin larvae. J Struct Biol 148:123-30. doi:10.1016/j.jsb.2004.04.001

Shimizu K, Noro N, Matsuda R (1988) Micromere differentiation in the sea urchin embryo: expression of primary mesenchyme cell specific antigen during development. Dev Growth Differ 30:35-47. doi:10.1111/j.1440-169X.1988.00035.x

Shimizu-Nishikawa K, Katow H, Matsuda R (1990) Micromere differentiation in the sea urchin embryo: 
immunochemical characterization of primary mesenchyme cell-specific antigen and its biological

role. Dev Growth Differ 32:629-636. doi: 10.1111/j.1440-169X.1990.00629.x

Shiomi K, Yamaguchi M (2008) Expression patterns of three Par-related genes in sea urchin embryos.

Gene Expr Patterns 8:323-330. doi:10.1016/j.gep.2008.01.002

Suzuki A, Ohno S (2006) The PAR-aPKC system: lessons in polarity. J Cell Sci 119:979-987

Tabuse Y, Izumi Y, Piano F, Kemphues KJ, Miwa J, Ohno S (1998) Atypical protein kinase C cooperates with PAR-3 to establish embryonic polarity in Caenorhabditis elegans. Development $125: 3607-3614$

Watts JL, Etemad-Moghadam B, Guo S, Boyd L, Draper BW, Mello CC, Priess JR, Kemphues KJ (1996) par-6, a gene involved in the establishment of asymmetry in early C. elegans embryos, mediates the asymmetric localization of PAR-3. Development 122:3133-3140 


\section{Table 1}

Skeletal rod formation and gut differentiation following the elimination of Par6 function in chimeric larvae composed of PMC-less hosts and transplanted PMCs

\begin{tabular}{lccccccccc}
\hline \multirow{2}{*}{ Hosts (PMCs) } & \multicolumn{4}{c}{ Rod elongation } & Abnormal & Posterior & Tripartite & AP \\
& \cline { 2 - 4 } & AL & PO & T & B & rod patterns & connections & guts & activity \\
\hline N (N) & $13 / 13$ & $13 / 13$ & $13 / 13$ & $13 / 13$ & $3 / 13^{* 1}$ & $11 / 13$ & $13 / 13$ & $6 / 6$ \\
N (Mpar6) & $10 / 11$ & $11 / 11$ & $9 / 11$ & $11 / 11$ & $9 / 11$ & $0 / 11$ & $11 / 11$ & $3 / 3$ \\
Mpar6 (N) & $5 / 6$ & $2 / 6$ & $4 / 6$ & $6 / 6$ & $0 / 6$ & $5 / 6$ & $4 / 6$ & $0 / 4^{* 2}$ \\
\hline
\end{tabular}

${ }^{* 1}$ Two of the three larvae had a normal branching pattern, but formed extra rods.

${ }^{* 2}$ Larvae with tripartite gut were examined. 


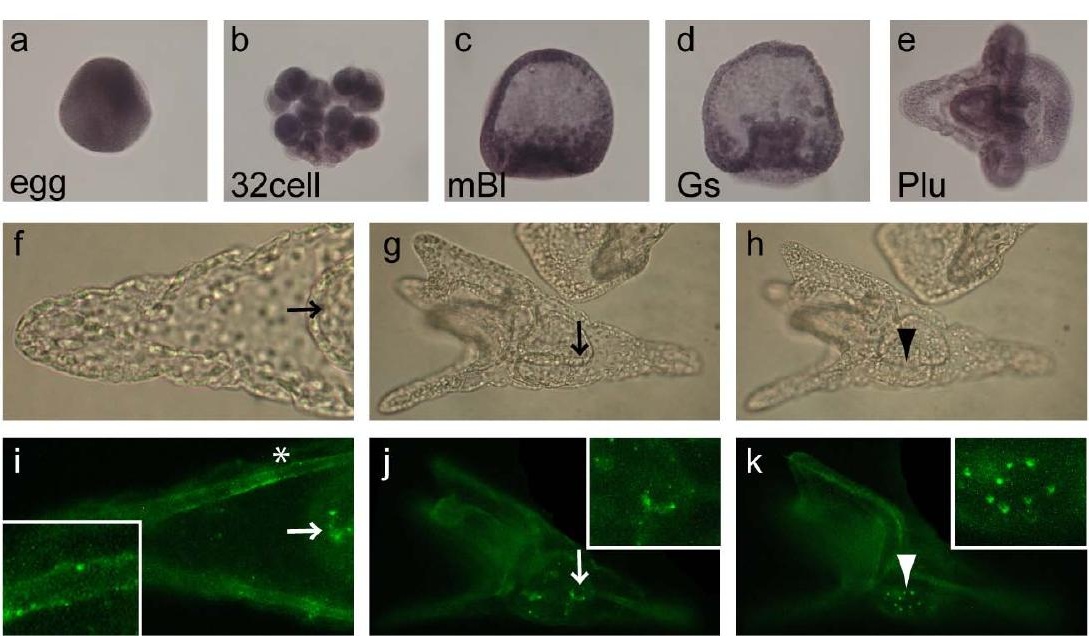




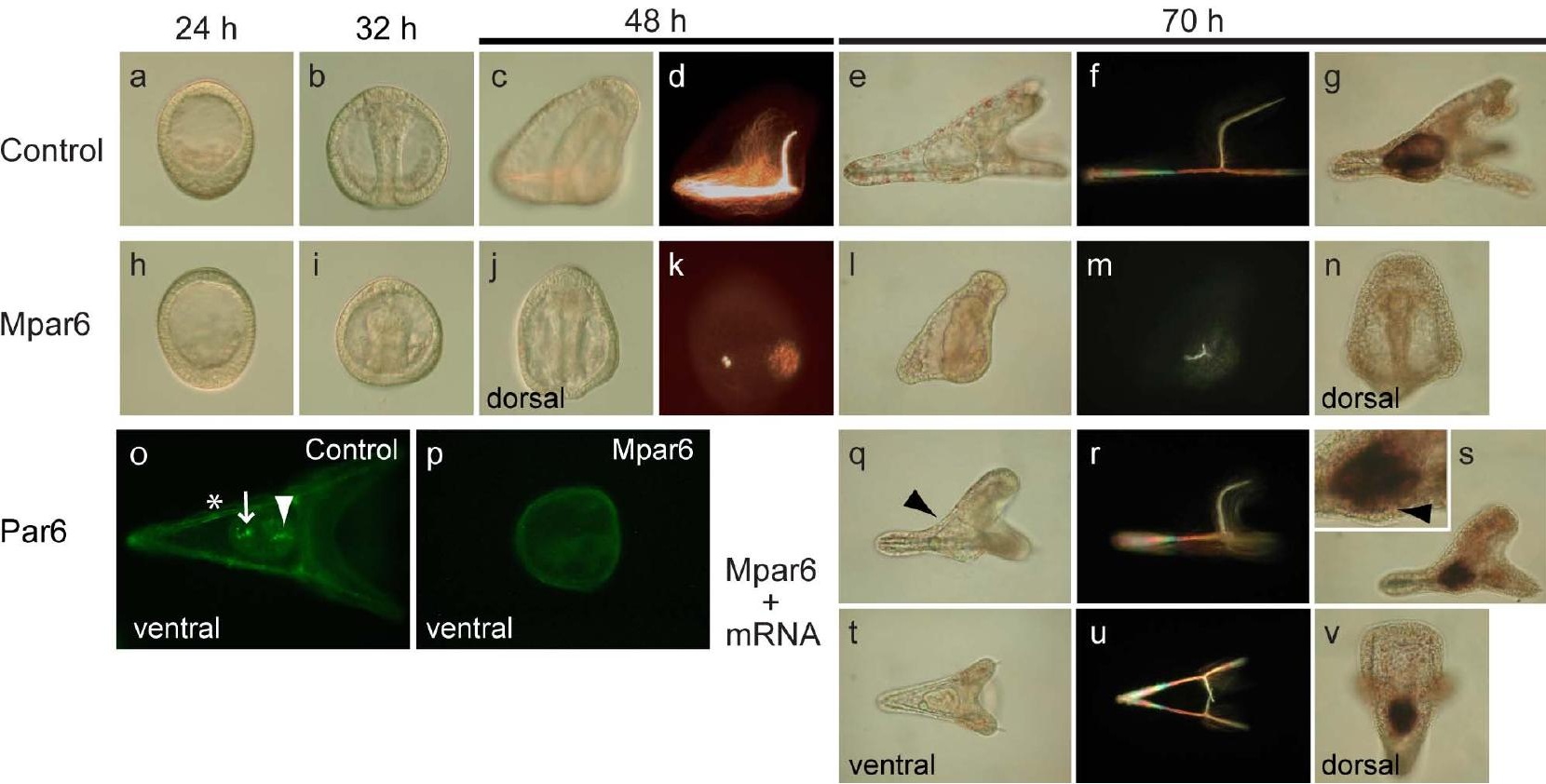


a

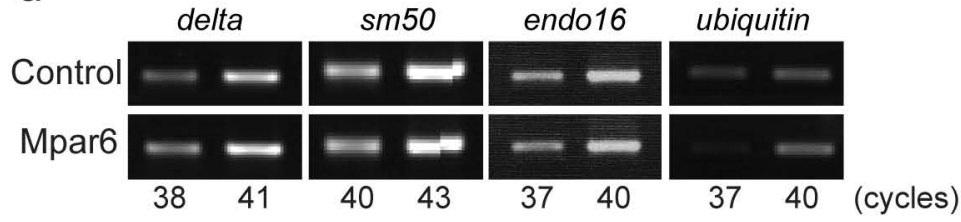

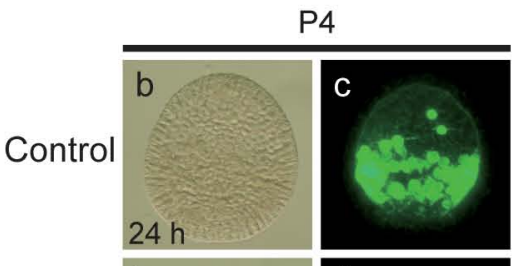

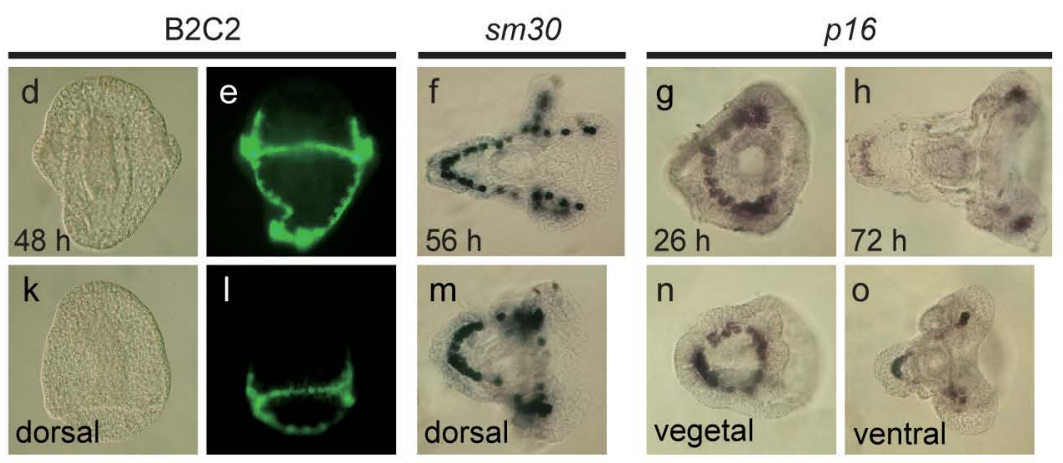

Mpar6

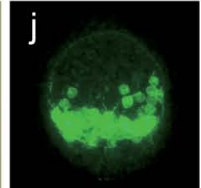


$48 \mathrm{~h}$

(6000) Normal (Normal)<smiles>C1=CC=CC=CC=1</smiles>
Normal (Mpar6)

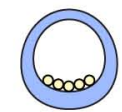
Mpar6 (Normal)

$\mathrm{s}$

AP activity
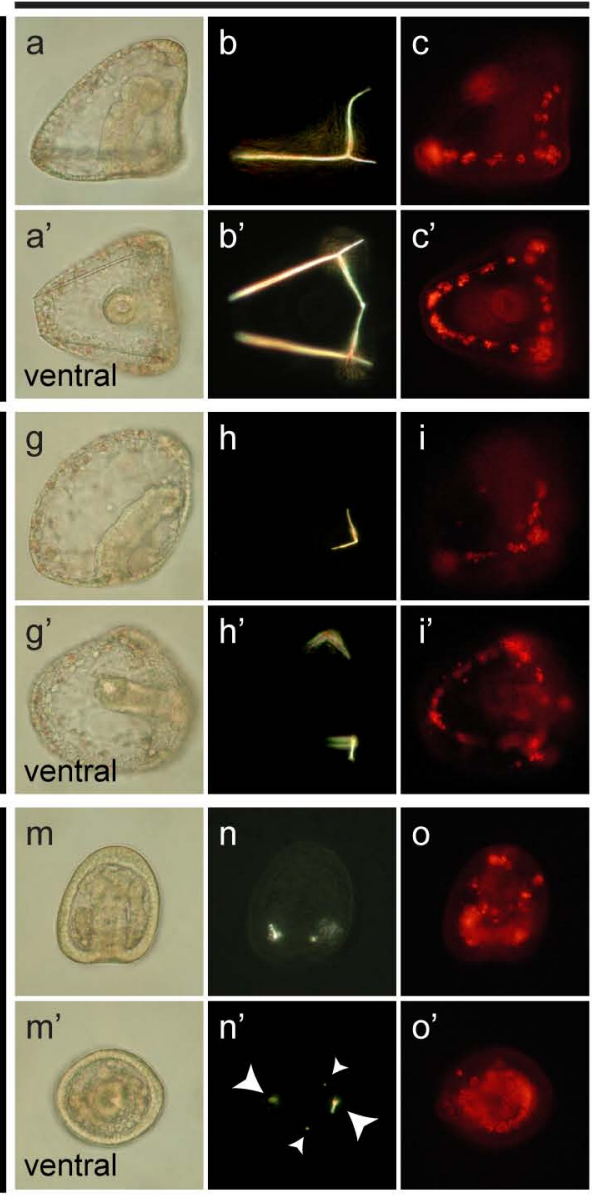

ventral
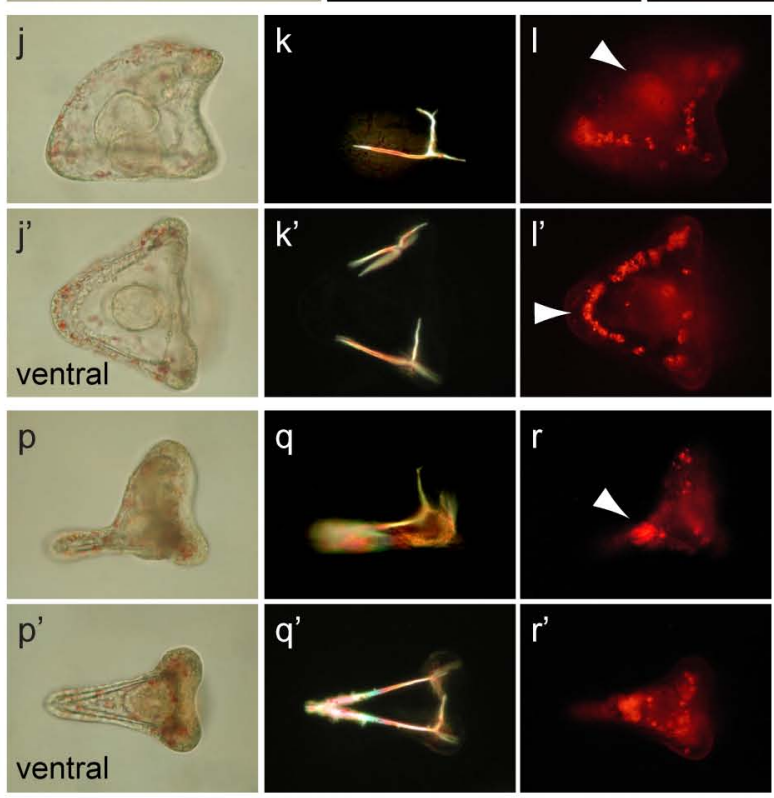

t
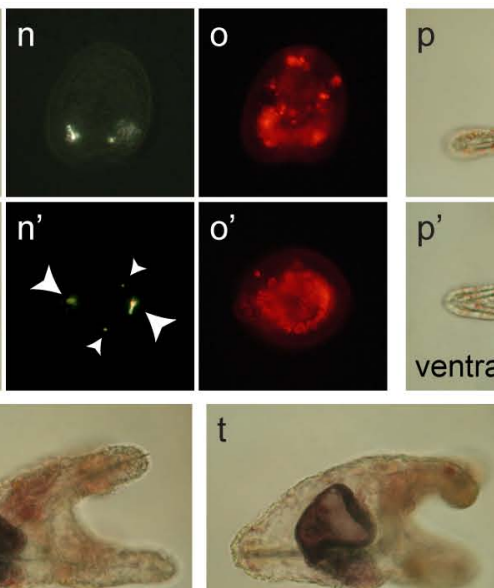

Normal (Mpar6)

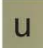

u

Mpar6 (Normal)

$72 \mathrm{~h}$ 
$22 \mathrm{~h} \quad 26 \mathrm{~h} \quad 52 \mathrm{~h}$

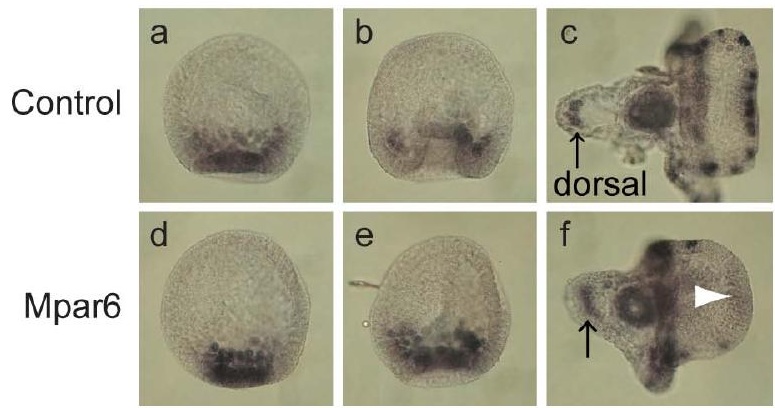


Fig. S1 Co-injection of a lower dose of mRNA injection ( 10 pg/egg) rescues Mpar6 larvae (a, a') from defects in skeletogenesis and gut differentiation, but not from dwarfish ectoderm patterning (b, b’). In contrast, injection of Par6 mRNA ( 15pg/egg) does not affect the skeletal patterning (c, c', d, d')

Fig. S2 Skeletal rod formation in eleven chimeric larvae composed of PMC-less hosts and PMCs including Mpar6. The elongation and pattern of skeletal rods are summarized in Table 1

Fig. S3 Skeletal rod formation in six chimeric larvae composed of PMC-less hosts including Mpar6 and normal PMCs. The elongation and pattern of skeletal rods are summarized in Table 1

Fig. S4 Distribution of aPKC protein in larvae $(72 \mathrm{~h})$ analyzed by immunostaining according to the method of Prulière et al. (2011). The kinase is localized in the ciliary band ectoderm (a'-d'), coelomic sacs (b'), stomach (c'), and intestine (d'). Few signals are detected in the skeletogenic cells; however, those at the vertex show relatively stronger signals (e) 
a
$a^{\prime}$

b

C

d

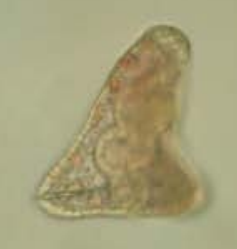


A1-1

left lateral

A1-2

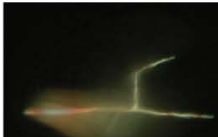

right lateral

right lateral

A2
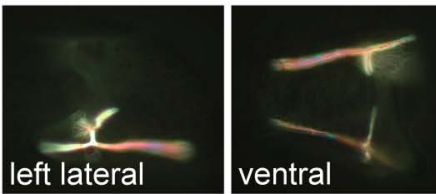

A3-1

A3-2
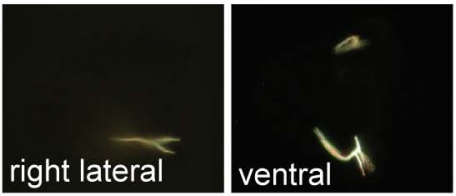

right lateral

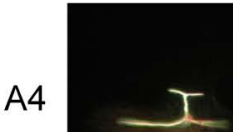

right lateral ventral
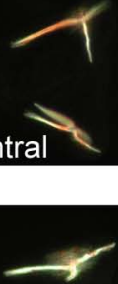

ventral
B1

right lateral

B2

right lateral
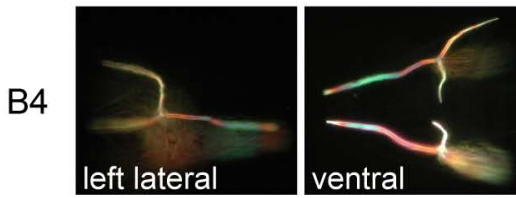

C4-1

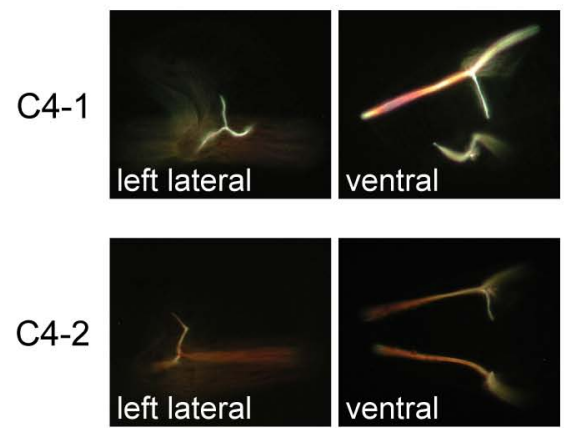

C4-2

left lateral
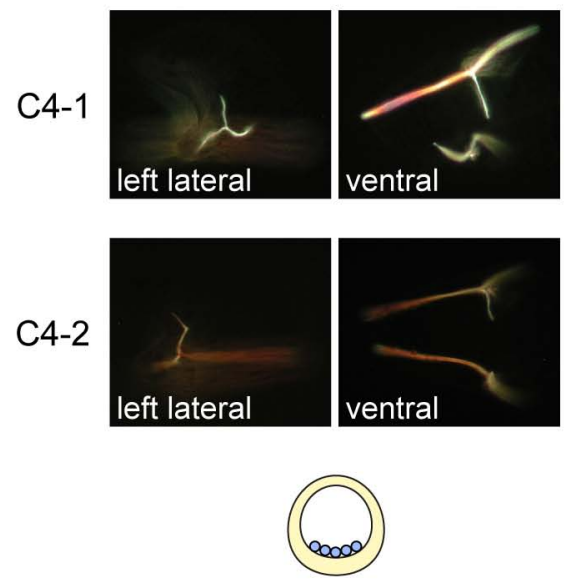

Normal (Mpar6) 


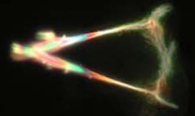

dorsal

A2

B1

left lateral

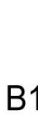

ventral
B2

Mpar6

(Normal)

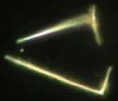

ventral dorsal

right lateral

B4

dorsal

B3

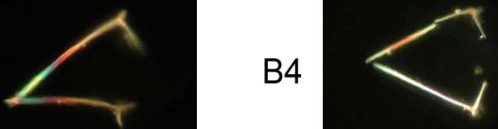

ventral

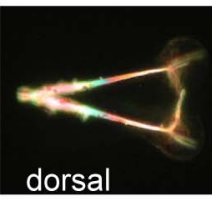

dorsal 


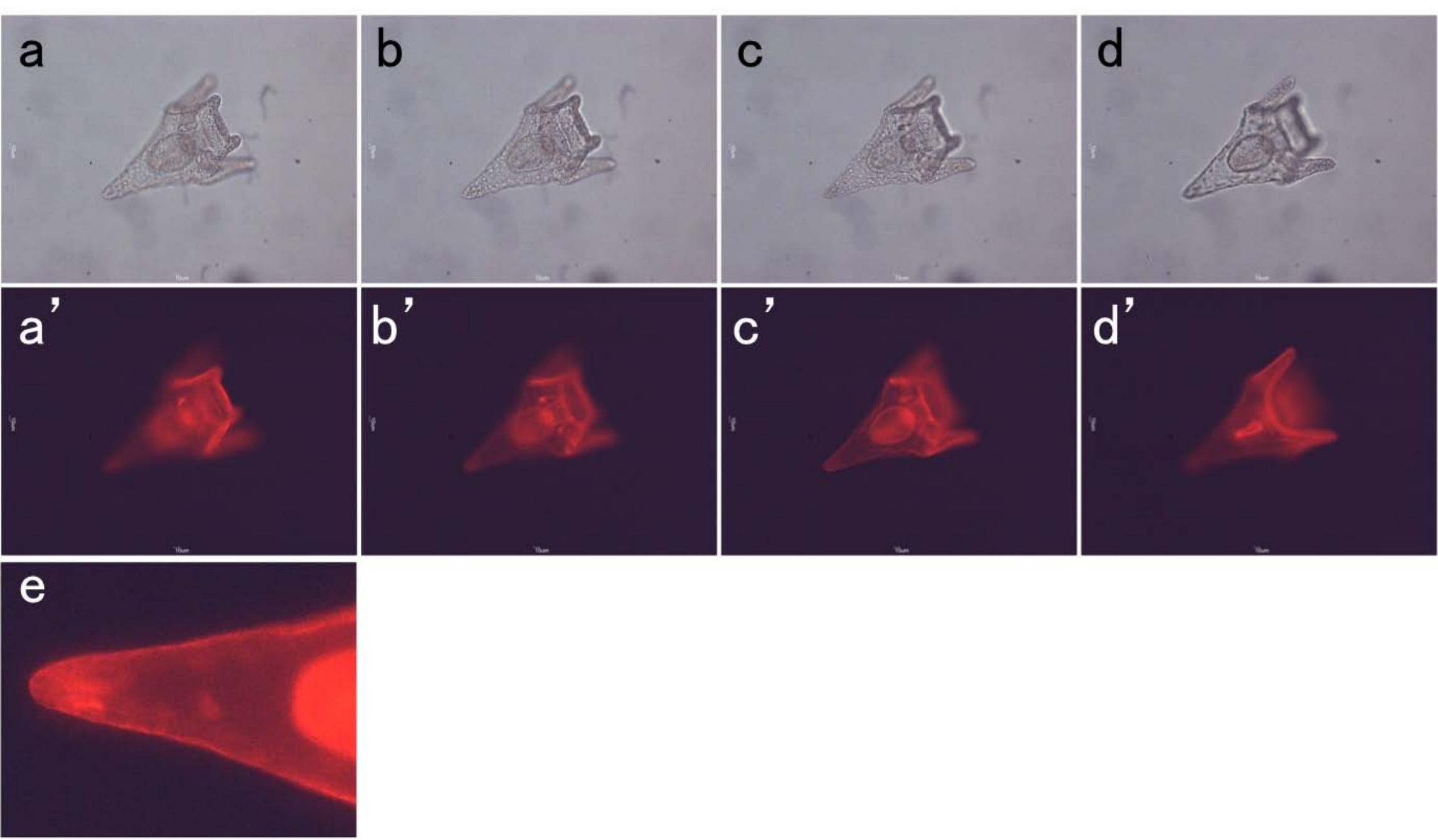

